\title{
LA EROSIÓN HISTÓRICA DE LA PLAYA DE SA RÀPITA (S. MALLORCA) ${ }^{1}$
}

\author{
José Ángel Martín Prieto \\ Universitat de les Illes Balears \\ josean33@gmail.com \\ Francesc Xavier Roig Munar \\ Quatre Consultoria Ambiental \\ xiscoroig@gmail.com \\ Antonio Rodríguez Perea \\ Universitat de les Illes Balears \\ arperea2@gmail.com \\ Miquel Mir Gual \\ Universitat de les Illes Balears \\ miquel.mir@uib.es \\ Guillem Xavier Pons Buades \\ Universitat de les Illes Balears \\ guillemx.pons@uib.es \\ Bernardí Gelabert Ferrer \\ Universitat de les Illes Balears \\ bernardi.gelabert@uib.es
}

\section{RESUMEN}

La evolución morfosedimentaria de la playa de sa Ràpita (Sur de Mallorca) se ha visto afectada a partir de la construcción, en 1977, del puerto deportivo ubicado en su extremo NO. La consecuencia más importante ha sido la modificación de la deriva litoral, ya que el puerto actúa como trampa sedimentaria. Este hecho impide que el sedimento pueda distribuirse a lo largo de la playa, como ocurría antes de su construcción. La playa ha sufrido procesos erosivos importantes a partir de 1977 hasta la actualidad. Así, a partir de esa fecha, el sector más próximo al puerto deportivo ha aumentado su superficie con una media de $0.5 \mathrm{~m} / \mathrm{a}$, mientras que el resto de la playa se erosiona con una media de entre -0.19 y $-0.34 \mathrm{~m} / \mathrm{a}$. El estudio se ha realizado con la ayuda de un Sistema de Información Geográfica y con la herramienta Digital Shoreline Analysis System, que permite cuantificar los procesos erosivos a lo largo de un periodo comprendido entre los años 1956 y 2012.

Palabras clave: Mallorca; sa Ràpita; deriva litoral; erosión; GIS; DSAS.

\section{ABSTRACT}

\section{Historical beach erosion of sa Ràpita (S. Mallorca)}

The morphosedimentary evolution of sa Rapita beach (South of Mallorca) has been altered by the construction, in 1977, of a marina located to the NW. The most important consequence has been the

\footnotetext{
1 Este artículo se ha beneficiado de la ayuda del proyecto "Crisis y reestructuración de los espacios turísticos del litoral español" (CS0201564468-P) del Ministerio de Economía y Competitividad (MINECO) y del Fondo Europeo de Desarrollo Regional (FEDER).
} 
alteration of the littoral drift, as the port acts as a sediment trap. This inhibits the distribution of the sediment along the beach. The beach has suffered significant erosion from 1977 to the present. From this date, the marina sector has increased its surface by an average of $0.5 \mathrm{~m} / \mathrm{a}$, while the rest of the beach has been eroded on average between -0.19 and $-0.34 \mathrm{~m} / \mathrm{a}$. The study was conducted with the help of a Geographic Information System and the Digital Shoreline Analysis System tool, to quantify erosive processes over a period between 1956 and 2012.

Keywords: Mallorca; sa Ràpita; littoral drift; erosion; GIS; DSAS.

\section{INTRODUCCIÓN}

La vulnerabilidad del litoral, así como los cambios de la línea de costa e inundaciones, afectan a la mayoría de las costas de todo el mundo, siendo responsables de la destrucción de propiedades e infraestructuras. Los cambios en el litoral a corto y largo plazo se asocian con varios factores, tales como el suministro de sedimentos, transporte litoral, cambios del nivel del mar, la influencia antrópica, la hidrodinámica del entorno cercano a la costa, las tormentas y la naturaleza de los accidentes geográficos costeros (Nordstrom, 2000; Kumar \& Jayappa, 2009). Por lo tanto, la comprensión de las posiciones de línea de la playa y la tendencia de erosión/acreción a través del tiempo, son de elemental importancia para científicos, ingenieros y gestores (Douglas \& Crowell, 2000; Boak \& Turner, 2005).

En el presente año, se cumple el vigésimo aniversario de la publicación en la IV Reunión de Geomorfología sobre la evolución de la línea de costa de la playa de sa Ràpita (Servera \& Martín, 1996). Este trabajo fue uno de los primeros en el archipiélago Balear en que se hacía referencia al comportamiento morfodinámico de una playa asociada a la construcción de una gran infraestructura costera, en este caso un puerto deportivo en uno de sus extremos.

Las conclusiones más importantes a las que se llegó en el citado trabajo fueron: la alteración de la dinámica sedimentaria de la playa como consecuencia directa de la construcción del puerto deportivo en su extremo noroccidental, produciendo un giro de la playa, al alterarse la hidrodinámica marina, con un incremento notable de su superficie en el sector más próximo al puerto y erosión en el resto. También se puso de manifiesto, el impacto que produce la limpieza de la playa (retirada de restos de Posidonia oceanica depositada sobre la arena) con maquinaria pesada y, por último, el impacto masivo de los usuarios que pisotean la duna delantera facilitando la erosión.

La principal diferencia entre este trabajo y el de Servera \& Martín (1996) reside en la metodología utilizada. El contraste es importante, ya que entonces se realizó la cartografía a partir de ajustar toda la fotografía aérea (1968, 1973, 1983 y 1990) a una escala 1.5000, siendo posteriormente digitalizados en un software de diseño gráfico. Los errores más importantes se centraron en el proceso de identificación de las posiciones de la línea de costa y su cálculo posterior. Finalmente, se añadió un levantamiento topográfico para el último año de estudio (1994). Sin embargo, la diferencia más importante reside en la utilización de un Sistema de Información Geográfica [SIG].

A pesar de que el uso de las fotografías aéreas cuenta con algunas desventajas, la fotogrametría aún representa una herramienta muy útil a la hora de delimitar la posición de la línea de costa con altos niveles de precisión. Además, si se cuenta con el software requerido para facilitar su procesamiento y posterior análisis, resultan baratas y fáciles de adquirir comparado con otros métodos (Ojeda, Vallejo, Hernández \& Álvarez, 2007; Virdis, Oggiano \& Disperati, 2012).

Un SIG constituye un excelente instrumento para el análisis e integración de datos debido a su capacidad para identificar las conexiones espaciales entre las diferentes capas de información. De esta manera, es posible construir modelos para la evolución geomorfológica y predecir los cambios en las zonas costeras, siendo parte fundamental en el análisis de la evolución de los estudios costeros (Rodríguez, Montoya, Sánchez \& Carreño, 2009). Esto pone de relieve la evolución espacial y temporal de los procesos dinámicos, así como los factores que controlan su comportamiento con el fin de analizar escenarios, evaluar el impacto en los ambientes litorales y gestionar de manera adecuada (Zhang \& Grassle, 2002). Una de las consecuencias más importantes de este proceso, es el poder incorporar el análisis cuantitativo en la evolución de los sistemas litorales, así como la modelización especializada de algunos procesos claves en geomorfología litoral, ya que, debido a su elevado dinamismo a escala humana (playas, dunas, etc...) y a su impacto sobre muchas actividades antrópicas (turismo, espacios urbanizados, etc...), es necesario un continuado control geométrico de las formas resultantes (Ojeda, et al., 2007). 
Una vez que los datos se incorporan al SIG, cualquier análisis espacial y temporal puede ser generado. Herramientas como el Digital Shoreline Analysis System [DSAS] y los métodos de captura de datos se pueden utilizar para producir representaciones del terreno de calidad. El conjunto de datos, una vez recopilado puede ser utilizado para realizar cualquier cálculo, incluyendo las tasas de erosión-acreción, evolución de dunas, el transporte de sedimentos, etc.

Esta herramienta junto a la creación y desarrollo de las Infraestructuras de Datos Espaciales [IDE], ha permitido y facilitado el trabajo referente a la ortorectificación y la digitalización, minimizando los posibles errores propios de la georreferenciación. Una Infraestructura de Datos Espaciales es un sistema informático integrado por un conjunto de recursos (catálogos, servidores, programas, aplicaciones, páginas web,.. ) que permite el acceso y la gestión de conjuntos de datos y servicios geográficos (descritos a través de sus metadatos), disponibles en Internet, que cumple una serie normas, estándares y especificaciones que regulan y garantizan la interoperabilidad de la información geográfica (http://www.idee.es/).

\section{1. Área de estudio}

La playa de sa Ràpita se encuentra situada en el litoral meridional de la isla de Mallorca (figura 1). Se trata de una playa arenosa que se extiende desde el puerto deportivo del mismo nombre, situado en su extremo noroccidental, hasta el promontorio de ses Covetes en el extremo sudeste, con una longitud aproximada de $1.3 \mathrm{~km}$ y una orientación del NO al SE. La playa se ve interrumpida por la presencia de dos promontorios rocosos, que son referencia para la fraccionar de la playa en tres sectores (sector del puerto, central y de ses Covetes), formados por eolianitas y arenas de playa cementadas que pertenecen al Pleistoceno superior (Vicens, Morey \& Pons, 2006).

Desde la playa hacia tierra, se extiende en dirección NE un sistema dunar. Desde el punto de vista de su clasificación morfodinámica, la playa de sa Ràpita, por similitud con la vecina playa de es Trenc (a tan solo $0.6 \mathrm{~km}$ de distancia) puede ser incluida en un estadio intermedio (Wright \& Short, 1983). Este tipo morfodinámico corresponde a playas semi-cerradas, normalmente con un sistema dunar que se desarrolla hacia tierra (Gómez, et al., 2007). Estos autores destacan que en la estación invernal suelen migrar a un estado reflectivo, mientras en la época estival hacia un estado más disipativo. En las fotografías aéreas de los años 1968, 1981, 1989, 1995 y 2006 se puede distinguir barras sumergidas de tipo crescentic y paralelas a la línea de costa.

Figura 1. Localización del área de estudio.

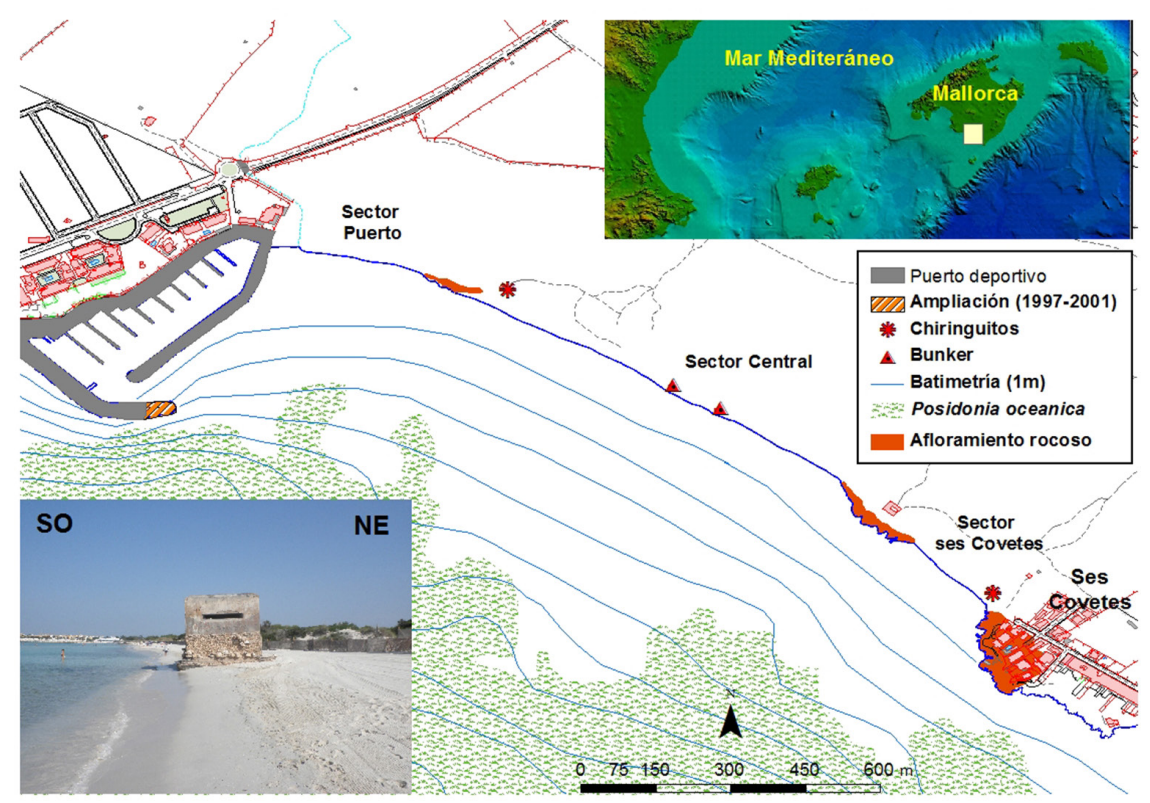

En la parte inferior izquierda, se puede apreciar uno de los bunkers (fotografía de julio de 2010) situados en el centro de la playa y la erosión de la duna delantera.

Elaboración propia. 
En cuanto a la petrología de su sedimento, la composición es predominantemente bioclástica (98\%), siendo el resto litoclastos, en su mayor parte calcáreos. Su textura se caracteriza por un tamaño de grano que oscila entre fino y medio. Este material se distribuye preferentemente a ambos extremos de la playa, mientras que en su parte central se pueden observar texturas tipo grava y guijarros, como consecuencia del desmantelamiento erosivo de eolianitas y de una playa fósil (Servera \& Martín, 1996; Vicens, et al., 2006). Su contenido en $\mathrm{CaCO}^{3}$ se sitúa sobre el 80\% (Jaume \& Fornós, 1992).

Por lo que respecta al Puerto Deportivo de sa Ràpita, fue construido entre los años 1974 y 1977 en el extremo noroccidental de la playa. Posteriormente, el muelle meridional fue ampliado en $40 \mathrm{~m}$ entre los años 1998 y 2001 (figura 1).

En el centro de la playa se encuentran dos bunkers construidos a partir de 1939 (Albertí, 1998), que nos permiten observar a simple vista la pérdida de superficie de la misma (figura 1). El objetivo de estas estructuras rígidas, distribuidas a lo largo de la costa, era la de no ser vistos desde el mar, por lo que eran construidas entre las dunas delanteras. Por tanto, constituyen un magnífico elemento para estimar la erosión que ha tenido lugar, no solo en la playa de sa Ràpita, si no en otras como la vecina playa de es Trenc (Martín, Roig, Rodríguez \& Pons, 2010) a lo largo de las últimas décadas.

Por lo que respecta a la batimetría, la isobata de $-10 \mathrm{~m}$ se encuentra a $900 \mathrm{~m}$ de la costa y la de $-20 \mathrm{~m}$ a una distancia cercana a los $2.000 \mathrm{~m}$. Esto significa que las olas de alta energía tocan fondo y comienzan a modificar sus características a una distancia considerable de la playa. Por otra parte, la isobata de -1 m se encuentra a los $80 \mathrm{~m}$ de la línea de costa y la de $-2 \mathrm{~m}$ a más de $150 \mathrm{~m}$ de la orilla. En definitiva, podemos decir que las olas que modifican los rasgos morfológicos de este litoral se producen principalmente en ocasión de grandes temporales. Este aspecto es importante a la hora de interpretar los procesos sedimentarios, los cuales dependen más directamente de la deriva litoral (figura 2) que de los efectos directos del oleaje (Servera \& Martín, 1996).

El otro aspecto destacado de la playa sumergida es la presencia de Posidonia oceanica, fanerógama marina conocida popularmente como «alga». Se desarrolla formando una pradera, la cual crece principalmente a partir de una bio-estructura hecha de raíces, rizomas y hojas, conocida como "mata". Su presencia juega un papel muy importante en la dinámica natural. Por una parte, al desarrollarse en el nearshore en una profundidad que oscila entre los 2 y $40 \mathrm{~m}$, sirve como atenuante de la altura de las olas más altas, con lo que la profundidad relativa disminuye (Elginoz, Kabdaslit \& Tanik, 2011; Sánchez, Sánchez \& Memos, 2011; Stratigaki et al., 2011). Además, sus rizomas fijan y estabilizan el sedimento en el nearshore (Gacia \& Duarte, 2001; Manca, et al., 2012; Terrados \& Duarte, 2000). Por lo que respecta a la parte emergida, los restos de hoja de Posidonia actúan como una efectiva defensa de la playa (Simeone $\&$ De Falco, 2013), ya que comienzan a acumularse sobre la playa en otoño con los primeros temporales. De esta forma, la playa subaérea está protegida del oleaje de alta energía, manteniendo un perfil estable (Cancemi \& Duron, 2008; De Falco, Baroli, Simeone \& Pergalini, 2002; Roig \& Martín, 2005). Esta acumulación denominada "banquette", es retirada de manera sistemática con maquinaria pesada a la parte posterior de la duna delantera por los gestores de la playa, con lo que en su extracción y transporte se pierde un volumen importante de sedimento que no retorna al balance sedimentario.

En el caso de la playa de sa Ràpita, la pradera se extiende aproximadamente desde la cota $-4 \mathrm{~m}$ hasta una profundidad aproximada de $-35 \mathrm{~m}$ (http://www.ideib.cat). Su límite superior muestra irregularidades, pero presenta continuidad a lo largo de la costa y a partir de la comparación visual de la fotografía aérea, no se ha observado retroceso aparente del mismo (figural).

\subsection{Clima marítimo}

Por lo que respecta a la dinámica marina de la zona de estudio ésta, al igual que el resto del ámbito Balear, apenas experimenta intensidad mareal, donde las oscilaciones debidas a la presión atmosférica son incluso más influyentes que la propia marea. En ese sentido, la importancia de las mareas es muy poco significativa, con valores que oscilan en torno a los $0.3 \mathrm{~m}$ (http://www.puertos.es).

Por lo que hace al oleaje, éste está condicionado por un fetch reducido, con un máximo de $700 \mathrm{~km}$ para el SSO y un mínimo de $280 \mathrm{~km}$ para el SE. Los datos de oleaje (Altura de ola significante y dirección) son proporcionados por Hindcast of Dynamic Processes of the Ocean and Coastal Areas of Europe [HIPOCAS], que es una base de datos de alta resolución temporal y espacial para las zonas costeras de Europa (Ratsimandresy, Sotillo, Carretero, Álvarez \& Hajji, 2008). Los datos de oleaje han sido recogidos por horas a lo 
largo de 44 años, durante el periodo comprendido entre 1958-2001, constituyendo la base de datos más completa para el Mediterráneo (Cañellas, 2010; Infantes, Terrados, Orfila, Cañellas \& Álvarez, 2009).

Figura 2. Rosas de oleaje entre abril-septiembre y octubre-marzo.

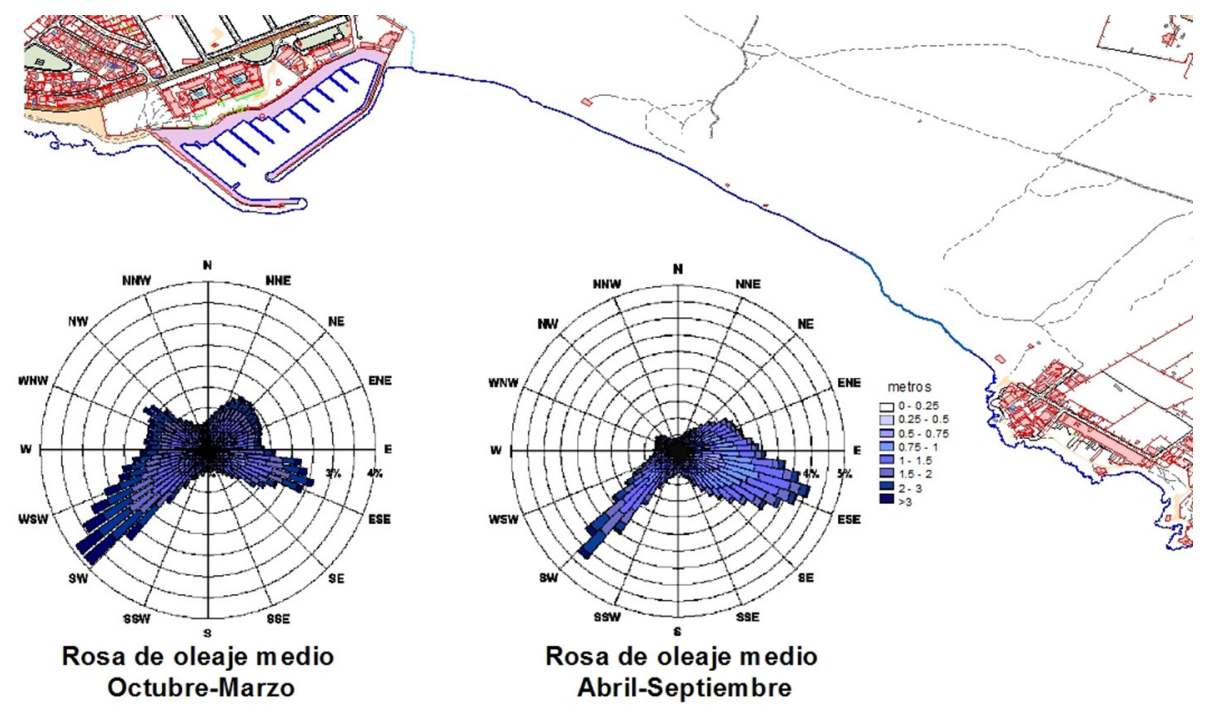

Fuente: Balaguer, et al., 2007.

Para este trabajo, se ha utilizado la base de datos del Nodo HIPOCAS 1694 (Balaguer, et al., 2007), localizado a unos $27 \mathrm{~km}$ al sureste de la zona de estudio (figura 2). La distribución temporal de la altura significante y su dirección, muestra una bimodalidad que ayuda a interpretar los procesos morfosedimentarios que tienen lugar en la playa. Por un lado, tenemos la rosa de oleaje correspondiente a los meses abril a septiembre, que presenta una dirección procedente del ESE, que favorece el transporte de sedimento hacia el oeste y una segunda dirección en sentido contrario, del SO, pero de menor frecuencia e intensidad. Entre los meses de octubre a marzo, la procedencia del oleaje, tiene una componente predominante del SO que presenta una mayor frecuencia e intensidad; de este modo, el sedimento es transportado de nuevo hacia el SE.

Por tanto, tenemos un transporte de sedimento hacia ambos lados de la playa, que será determinante para poder interpretar los procesos sedimentarios que tienen lugar antes y después de la construcción del puerto deportivo. En cuanto a Hs, el 73\% tiene una altura inferior a $1 \mathrm{~m}$, mientras la Hmx registrada desde 1958 tuvo lugar el 1 de diciembre de 1959, con una altura máxima de $7.2 \mathrm{~m}$, un periodo de $11.2 \mathrm{~s}$ y una dirección procedente del SO (http://www.puertos.es). La Hs50 para este nodo HIPOCAS, se estima en $8.76 \mathrm{~m}$ (http://www.costabalearsostenible.com).

\subsection{Objetivos}

En primer lugar, se trata de analizar la evolución histórica de la línea de costa de la playa de sa Ràpita en un periodo comprendido entre los años 1956 y 2012. En segundo lugar, profundizar en las causas que la han llevado a la situación regresiva actual. El tercer objetivo de este trabajo consiste en validar los supuestos planteados en el trabajo de Servera y Martín (1996), cuantificando los procesos erosivos posteriores a la publicación del citado trabajo. Finalmente, el último objetivo planteado es el de intentar predecir la tendencia de la playa con la finalidad de facilitar los posibles planes de gestión de la misma.

\section{METODOLOGÍA}

\subsection{Definición de la línea de costa}

Podemos definir la línea de costa como el umbral entre la masa de agua estabilizada del mar y la tierra firme continental (Dolan, Hayden, May \& May, 1980). La posición de la misma en un sistema playa-duna es altamente variable a corto plazo, donde los procesos de erosión y acumulación dentro de la dinámica 
sedimentaria pueden dar lugar a sustanciales variaciones de perfil y posición en cuestión de horas (Servera \& Martín, 1996). Según Elliot \& Clarke (1989) esta naturaleza dinámica de la línea de costa en períodos cortos de tiempo puede enmascarar la percepción de la evolución a largo plazo, ya que en ocasiones estos cambios pueden ser cíclicos y por lo tanto pueden dar lugar a resultados erróneos. Además estos mismos autores consideran necesario la observación de periodos largos, superiores a 10 años, a fin de realizar un diagnóstico fiable sobre el comportamiento evolutivo de la línea de costa (Boak \& Turner, 2005; Pajak \& Leatherman, 2002).

La línea de costa empleada para la digitalización ha sido el límite húmedo/seco. Esta línea marca el comienzo de la playa alta o playa seca (Ojeda, 2000). Para poder definir y digitalizar la línea de costa sobre la fotografía aérea, se ha estimado como más adecuado trabajar con el punto de máxima llegada del oleaje sobre la playa seca. La justificación para utilizar este límite se debe a la ausencia de mareas en el ámbito, con un rango que es casi desestimable (Martín, et al., 2010).

Esta marca o línea es visible en la mayoría de las fotografías (tanto las de color como las de blanco y negro), ya que el contraste entre seco y mojado suele definir una línea continua fácil de seguir a lo largo de la playa. Además de esto, la acumulación de hojas muertas de Posidonia oceanica depositadas sobre la playa constituye también un buen indicador para definir la línea de costa.

\subsection{Cartografía disponible y digitalización de la línea de costa}

Para medir sistemáticamente las variaciones en los cambios de la línea de costa a lo largo de un período suficientemente largo de tiempo, se ha establecido una metodología desarrollada a partir de la utilización conjunta de las ortofotos disponibles obtenidas de la "Infraestructura de Dades Espacials de les Illes Balears [IDEIB] (http://www.ideib.cat)", correspondientes a los años 1956, 1989, 2002, 2006, 2008, 2010 y 2012, conjuntamente con las fotografías aéreas verticales de los años 1968, 1973, 1979, 1981, 1983 y 1995 (tabla 1). Estas últimas han sido digitalizadas y posteriormente georreferenciadas con un SIG. Una vez digitalizadas las diferentes líneas de costa, todo el conjunto, se ha analizado con la ayuda de una herramienta llamada Digital Shoreline Analysis System [DSAS] (Thieler, Himmelstoss, Zichichi \& Ergul, 2009) (figura 3). Esta extensión constituye una herramienta muy práctica para llevar a cabo estudios de los cambios en la línea de costa, ya que a partir de una línea base, genera una serie de transectos a lo largo de la playa (un total de 142), con el intervalo espacial elegido, que para nuestro caso es de 10 $\mathrm{m}$, que cortan las diferentes líneas de costa digitalizadas. A partir de esa base, la herramienta ofrece varias estadísticas fundamentales para el análisis de la zona de estudio (Douglas \& Crowell, 2000; Thieler \& Danforth, 1994):

Tabla 1. Fechas de las ortofotos y fotografías aéreas utilizadas

\begin{tabular}{cccccc} 
Año & Tipo & Fecha & RMS & Resol. $(\mathrm{m})$ & Escala \\
\hline 2012 & Ortofoto & Mayo & & 0.25 & 1.5000 \\
2010 & Ortofoto & Noviembre & & 0.25 & 1.5000 \\
2008 & Ortofoto & Junio & & 0.5 & 1.5000 \\
2006 & Ortofoto & Junio & & 0.5 & 1.5000 \\
2002 & Ortofoto & Octubre & & 0.5 & 1.5000 \\
1995 & Georref. & Junio & 1.1 & 0.5 & 1.15000 \\
1989 & Ortofoto & Octubre & & 0.5 & 1.5000 \\
1983 & Georref. & Julio & 1.24 & 0.75 & 1.12000 \\
1981 & Georref. & Marzo & 1.16 & 1.1 & 1.15000 \\
1979 & Georref. & Febrero & 0.68 & 1.1 & 1.18000 \\
1973 & Georref. & Marzo & 0.4 & 1.1 & 1.14500 \\
1968 & Georref. & Junio & 1.1 & 1.1 & 1.18000 \\
1956 & Ortofoto & Julio & & 0.5 & 1.5000 \\
\hline
\end{tabular}

Elaboración propia. 
- Shoreline Change Envelope (SCE): Muestra la distancia entre las líneas más alejadas entre sí para cada transecto. Indica el dinamismo de la línea de costa sin tener en cuenta las fechas. Representa una distancia en metros, no una ratio (Thieler, et al., 2009).

- Net Shoreline Movement (NSM): Muestra la distancia entre las líneas de costa más antigua y más reciente, sin tener en cuenta si coinciden o no con las líneas más distantes entre sí. Al igual que SCE, representa una distancia en metros, no una ratio.

- End Point Rate (EPR): Muestra el valor del NSM, dividido por el número de años transcurridos en cada periodo, de modo que es un índice o tasa anual de movimiento en metros/año. Su principal ventaja es la facilidad de cálculo, siendo muy útil para estudiar distintas tendencias dentro de un periodo general de estudio. Su mayor desventaja es que al utilizar solo dos fechas se ignora información adicional como magnitudes o tendencias cíclicas. Por ello, este parámetro es comparado con otros como la regresión lineal o la recta de regresión ponderada (Dolan, Fenster \& Olmes, 1991).

- Linear Regression (LRR): este parámetro es utilizado para capturar tendencias de cambios de la línea de costa en periodos largos de tiempo. En el cálculo de este método, la regresión lineal es calculada a través de la intersección de puntos de cada transecto y la pendiente constituye la tasa del cambio expresada en metros/año. Es considerado un método fiable para predecir futuras posiciones del litoral, puede revelar qué tipo de asociación existe en particular (a través del valor R) y cuál es el valor de la varianza de la variable dependiente (la posición de la línea de costa en una fecha determinada, que es la variable independiente) (Crowell, Leatherman \& Buckley, 1991; Douglas \& Crowell, 2000). Sin embargo, el método de regresión lineal es susceptible a los efectos de valores atípicos y también tiende a subestimar la velocidad de cambio en relación con otras estadísticas (Dolan, et al., 1991; Genz, Fletcher, Dunn, Frazer \& Rooney, 2007).

- Weighted Linear Regression (WLR): la recta de regresión ponderada puede ser utilizada para describir el comportamiento histórico de la línea de costa. La información puede ser utilizada para remarcar áreas con las mayores tasas de cambio como posibles zonas de mayor vulnerabilidad erosiva (Virdis, et al., 2012). Es un parámetro estadístico importante, ya que tiene en cuenta las incertezas asociadas a los errores (especialmente en fotografías antiguas) como factor de ponderación en las ecuaciones de regresión. Este método se centra en los puntos de datos donde la exactitud de la posición litoral es inferior en un determinado año, con un intervalo de confianza del 95\% (Thieler, et al., 2009).

- Least Median of Squares (LMS): la mediana de los cuadrados es determinada por un proceso que calcula todos los valores posibles de la pendiente (la velocidad de cambio). Aunque el proceso de cálculo de la línea a los puntos de datos sigue la misma lógica que el método LRR, el método LMS es un estimador de regresión más robusto que minimiza la influencia de un valor atípico anómalo en la ecuación de regresión general (Thieler, et al., 2009). Sectores o líneas de costa identificadas como anómalas (outliers) por este método (por ejemplo por tormentas) son minimizados para su consideración en el procedimiento de alineación de la recta. Además, calcula todos los posibles valores de la pendiente (tasa de cambio).

- La Regresión lineal (LRR), la regresión lineal ponderada (WLR) y la mediana de los cuadrados (LMS) fueron seleccionadas porque se consideran estadísticamente más sólidas cuando hay un número importante de líneas de playa disponibles (Crowell, et al., 1991). También son las técnicas estadísticas más comúnmente aplicadas para expresar el movimiento de la costa y la estimación de las tasas de cambio (Thieler \& Danforth, 1994). Además, minimizan el potencial de error aleatorio y la variabilidad a corto plazo (cambios cíclicos) mediante el uso de un enfoque estadístico (Douglas \& Crowell, 2000).

Los valores positivos de los parámetros expuestos representan desplazamiento de la línea de costa hacia el mar (acreción), por el contrario, valores negativos indican desplazamiento hacia tierra (erosión). Así pues, con la ayuda de las fotografías aéreas y por medio de la comparación, se determina la tasa de cambio de la posición de la costa. En cuanto a la fiabilidad de los resultados para las tendencias y las tasas calculadas del cambio litoral, se determinan a partir de la incertidumbre, que representa los errores de medición asociados con los métodos de asignación de costas históricas. En este estudio los errores corresponden a las mediciones realizadas para determinar con la mayor exactitud posible cada posición de la costa (Pérez, Pires, Freitas, Rodrigues \& Chaminé, 2011). 
Figura 3. Diagrama mostrando los pasos necesarios para la obtención de los resultados

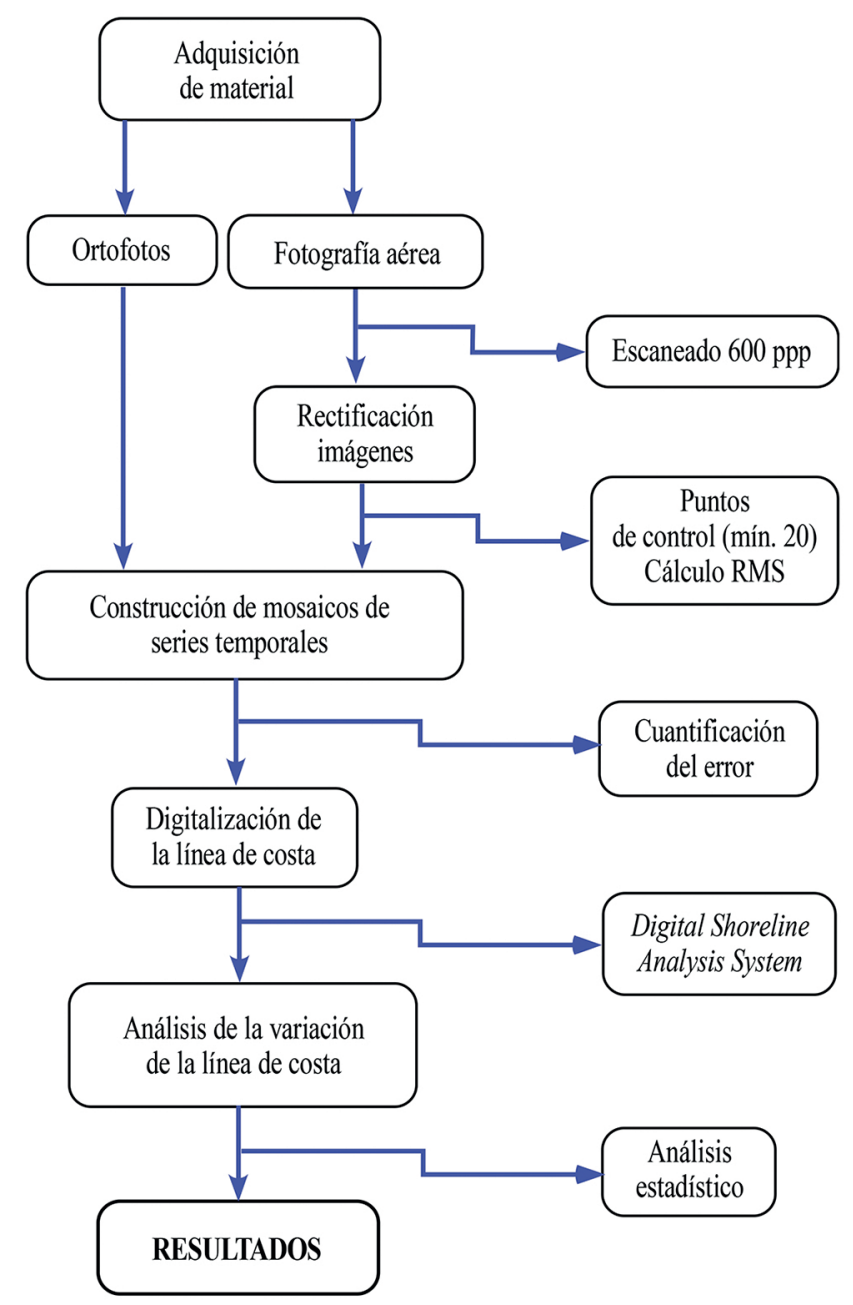

Elaboración propia.

\subsection{Errores e incertezas}

Para una óptima estimación de las tasas de cambio y reducir los efectos de la variabilidad temporal a escala de tiempo corta (mareas, tormentas, etc.), es necesario estimar las incertezas asociadas en cada fotograma a largo plazo (Ruggiero, et al., 2012). La evaluación del error o incertidumbre en estudios sobre la dinámica del litoral es un tema de suma importancia para proporcionar un marco adecuado para las tasas de cambio y para permitir el establecimiento de valores de umbral, por encima del cual el error sería significativo (Del Río \& Gracia, 2013). Debido a la generalmente amplia disponibilidad y bajo coste de los materiales, el análisis de fotografías aéreas verticales es la técnica más utilizada en los estudios de erosión costera, especialmente para la cuantificación de las tasas de recesión de la costa a medio plazo (Moore, 2000). Este uso implica tener en consideración diversas fuentes de error o incertidumbre tales como errores en los datos originales, errores de interpretación, errores de medida, etc. (Coyne, Fletcher \& Richmond, 1999; Morton, Miller \& Moore, 2004). La incerteza en este estudio ha sido calculada basándonos en el trabajo de Fletcher, Rooney, Barbee, Lim \& Richmond (2003) y Genz, et al., (2007). Se han manejado un total de tres variables utilizadas por dichos autores: el error en la digitalización, el error en la georreferenciación (rectificación) y el error en el escaneo, que representa la resolución de la imagen:

- Error en la digitalización (Ed): incorpora el error asociado con el proceso de digitalización de cada línea de costa. Para este trabajo se ha calculado teniendo en cuenta la desviación estándar a partir de varias digitalizaciones realizadas por el mismo especialista. 
- Error en la corrección geométrica (Eg): expresada como el error RMS del proceso (Morton, et al., 2004). Se calcula a partir del software y es la distancia entre los puntos de control establecidos sobre de la fotografía aérea y los puntos de control realizados sobre una ortofotogafía. Cabe señalar que la calidad del ajuste de las ecuaciones de transformación a los Puntos de Control expresada por el RMS no es completamente equivalente a la exactitud posicional de la imagen georectificada. Sin embargo, es una medida estadística que constituye una estimación ampliamente aceptada de la exactitud general del proceso (Hughes, McDowell \& Marcus, 2006). La medida de los errores a través del RMS además de su aceptación permiten su comparación con otros estudios (Jones, Hinkel, Arp, \& Eisner, 2008; Solomon, 2005).

- Error de escaneo o de píxel (Ep): la incertidumbre corresponde a los procesos de detección o de captura digital de las fotografías u ortofotografías originales. Se representa por el tamaño de píxel de la imagen (Catalao, Catita, Miranda \& Dias, 2002; Coyne, et al., 1999), ya que a mayor tamaño de los píxeles, mayor será la incertidumbre en la interpretación de la posición de la costa en la fotografía.

En este estudio no se incorpora la fluctuación mareal a los errores utilizados debido a que el efecto de las mareas en la zona de estudio es mínimo (Cowart, Walsh \& Corbett, 2010). Una vez que los tres componentes del error son calculados, la incerteza en la posición de la línea de costa de un determinado fotograma, es computada como la raíz cuadrada de la suma al cuadrado de cada componente (Fletcher, et al., 2003).

\section{RESULTADOS}

Uno de los aspectos más importantes para una correcta planificación y gestión costera, que necesita ser investigado, es la dinámica litoral. El análisis a largo plazo de la evolución costera utiliza datos históricos para identificar los sectores de la costa, donde la posición de la misma ha cambiado. Entre la información que puede ser obtenida de estos estudios, tenemos la tendencia general de la costa, ya sea erosionándose o creciendo y las tasas de erosión o acreción en cada lugar que se pueden utilizar para pronosticar posiciones de la costa futura (Arias, 2003).

Para poder dar respuesta a esos procesos, se ha analizado la evolución de la línea de costa de la playa de sa Ràpita a partir de su análisis por periodos de tiempo. Estos periodos se hallan condicionados por la construcción del puerto deportivo (y lógicamente por la disponibilidad de las fotografías aéreas), por tanto, un primer periodo es el comprendido entre 1956 y 1973, previo a su construcción y un segundo periodo se encuentra entre 1979 y 2012, posterior a la misma. Finalmente, se ha analizado el periodo global entre los años 1956 y 2012.

\subsection{Periodo comprendido entre 1956 y 1973}

Este periodo es anterior a la construcción del puerto deportivo, que se concluyó en 1977, por lo tanto estamos en un escenario donde la influencia antrópica sobre la playa es mínima, comparada con la actual. En general, la playa presenta acreción en los sectores Central y en el de ses Covetes, con valores medios de EPR de 0.37 y 0.5 m/a y NSM de 7.18 y 8.36 m respectivamente. Por el contrario, en el sector próximo al puerto hay erosión, con valor medio de EPR de -0.31 (m/a) y un NSM medio de -5.22 m (tabla 2 y figura 4).

Los valores de EPR y WLR son altamente concordantes a lo largo de este periodo (Figura 5), al igual que el SCE y NSM en el sector Central y de ses Covetes.

Tabla 2. Valores medios de EPR (m/a) y NSM (m) por periodos para los tres sectores

\begin{tabular}{lcccccccccc}
\hline & \multicolumn{2}{c}{$1956-73$} & \multicolumn{2}{c}{$1979-81$} & \multicolumn{2}{c}{$1981-83$} & \multicolumn{2}{c}{$1979-12$} & \multicolumn{2}{c}{$1956-12$} \\
\hline Sectores & EPR & NSM & EPR & NSM & EPR & NSM & EPR & NSM & EPR & NSM \\
\hline Puerto & -0.31 & -5.22 & 2.79 & 5.62 & 0.83 & 1.92 & 0.5 & 16.28 & 0.37 & 20.77 \\
Central & 0.37 & 7.18 & -0.35 & -0.71 & -0.7 & -1.61 & -0.19 & -6.34 & -0.17 & -9.34 \\
Ses Covetes & 0.5 & 8.36 & -1.32 & -2.49 & & & -0.34 & -11.18 & -0.17 & -9.35 \\
\hline
\end{tabular}

(En color rojo, los valores negativos)

Elaboración propia. 


\subsection{Periodo comprendido entre 1979 y 2012}

Es el periodo de tiempo posterior a la construcción del puerto deportivo (inaugurado en 1977), donde queda patente la influencia que su construcción tiene sobre la playa, con importantes alteraciones (figura 4, tabla 2).

La diferencia de este periodo con respecto al anterior es notable. En éste se aprecia como en los tres sectores se ha invertido la tendencia, donde antes había acreción, ahora hay erosión y viceversa.

Ello se puede apreciar en el rango del NMS, que ha aumentado notablemente. Así, en el sector Puerto, ha pasado de un valor negativo $(-5.22 \mathrm{~m})$ a alcanzar una media de acreción de $16.28 \mathrm{~m}$, con un valor EPR de $0.5 \mathrm{~m} / \mathrm{a}$. Por el contrario, el sector Central, retrocede ligeramente una media de $-6.34 \mathrm{~m}$, con EPR de $-0.19(\mathrm{~m} / \mathrm{a})$, mientras que el sector de ses Covetes se presenta como el más erosivo, con un retroceso medio de $-11.18 \mathrm{~m}$ y EPR de -0.34 (m/a) (figuras 4 y 5 , tabla 2).

Otra diferencia con el periodo anterior de 1956-1973 es visible en la figura 5, donde se puede observar el incremento del SCE, especialmente en los sectores Puerto y Central (se pasa de 6.08 y $8.75 \mathrm{~m}$ a 21.15 y 19 m de media entre 1979-2012). Hay una mayor variabilidad de la superficie de playa afectada con respecto al periodo anterior, que se aprecia por los valores de WLR y EPR, llegando a alcanzar un valor máximo de $29.57 \mathrm{~m}$, en el entorno más cercano al puerto.

Dentro de este periodo de análisis, que se extiende a lo largo de 33 años, puede analizarse la influencia del puerto inmediatamente después de su construcción, en un espacio de tiempo corto que comprende los periodos 1979-1981 y 1981-1983, con el objetivo de determinar la tasa de erosión-sedimentación, fundamentalmente en este sector Puerto.

\subsection{Periodos comprendidos entre 1979-1981 y entre 1981-1983}

El primero de los dos es el periodo analizado inmediatamente tras la construcción del puerto. Destacan en primer lugar las elevadas tasas de EPR en el sector Puerto (media de 2.79 m/a) y NSM de $5.62 \mathrm{~m}$. Por el contrario, los otros dos sectores presentan en este periodo valores negativos tanto en EPR (-0.35 y - $1.32 \mathrm{~m} / \mathrm{a}$ en el sector Central y ses Covetes respectivamente) como en NSM, con valores de -0.71 y -2.49 m (figura 6, tabla 2).

Estos valores se ralentizan en el siguiente bienio, reduciéndose EPR a $0.83 \mathrm{~m} / \mathrm{a}$ en el sector Puerto y NSM a $1.92 \mathrm{~m}$. Sin embargo, se incrementa la erosión en el sector Central con valores de -0.7 m/a y -1.61 m de EPR y NSM respectivamente, con una ralentización en el sector de ses Covetes (figura 6, tabla 2).

Por tanto, tales resultados muestran que el proceso de sedimentación y acreción en el sector Puerto se inicia de forma inmediata y con las tasas más elevadas entre 1979 y 1981 y una ralentización entre 1981 y 1983. Al mismo tiempo, los otros dos sectores comienzan a presentar procesos erosivos a la inversa, es decir, incrementándose en el periodo 1981-1983 (figura 6). Desde el momento de la construcción del puerto deportivo, los procesos acrecionarios están siempre presentes en el sector más próximo al mismo y erosivos en el resto de la playa.

\subsection{Periodo comprendido entre 1956 y 2012}

La dinámica de la playa en el conjunto de todos los fotogramas analizados, se puede observar en las figuras 4 y 5 . En la primera de ellas, se aprecia claramente el notable incremento de la superficie de la playa en el sector Puerto, con tasas máximas de $0.79 \mathrm{~m} / \mathrm{a}$ y $44.08 \mathrm{~m}$ en la zona más próxima al mismo, que disminuyen a medida que nos alejamos de él. La tasa media de EPR y NSM es de 0.37 m/a y 20.77 m respetivamente (tabla 2 ).

El otro aspecto que queda claro es que el resto de la playa se erosiona, con los valores máximos en el centro $(-0.35 \mathrm{~m} / \mathrm{a}$ y $-19.78 \mathrm{~m})$ y este del sector Central, con medias de $-0.17 \mathrm{~m} / \mathrm{a}$ y $-9.34 \mathrm{~m}$ de retroceso respectivamente. El sector de ses Covetes presenta máximos de $-0.32 \mathrm{~m} / \mathrm{a}$ y $-17.39 \mathrm{~m}$, con valores medios de $-0.17 \mathrm{~m} / \mathrm{a}$ y $-9.35 \mathrm{~m}$ respectivamente, prácticamente iguales al sector Central.

También se puede apreciar en la figura 5, como el SCE presenta valores elevados en toda la playa (especialmente en el sector del Puerto) comparados con el NSM, con medias de 29.81, 23.71 y 24.45 m para el sector Puerto, Central y ses Covetes respectivamente. Recordemos que este parámetro muestra la distancia entre las líneas más alejadas entre sí para cada transecto, por tanto indica el dinamismo de la línea de costa. 
También podemos señalar la alta correlación entre EPR y WLR en los sectores Puerto y ses Covetes. Esto no ocurre en el Central, donde el segundo parámetro presenta una medida más ponderada al analizar todas las líneas de costa, a diferencia del primero que analiza la diferencia entre la primera y la última.

Figura 4. EPR y NSM para el periodo 1956-1973, previo a la construcción del puerto deportivo, 1979-2012 y global entre 1956 y 2012

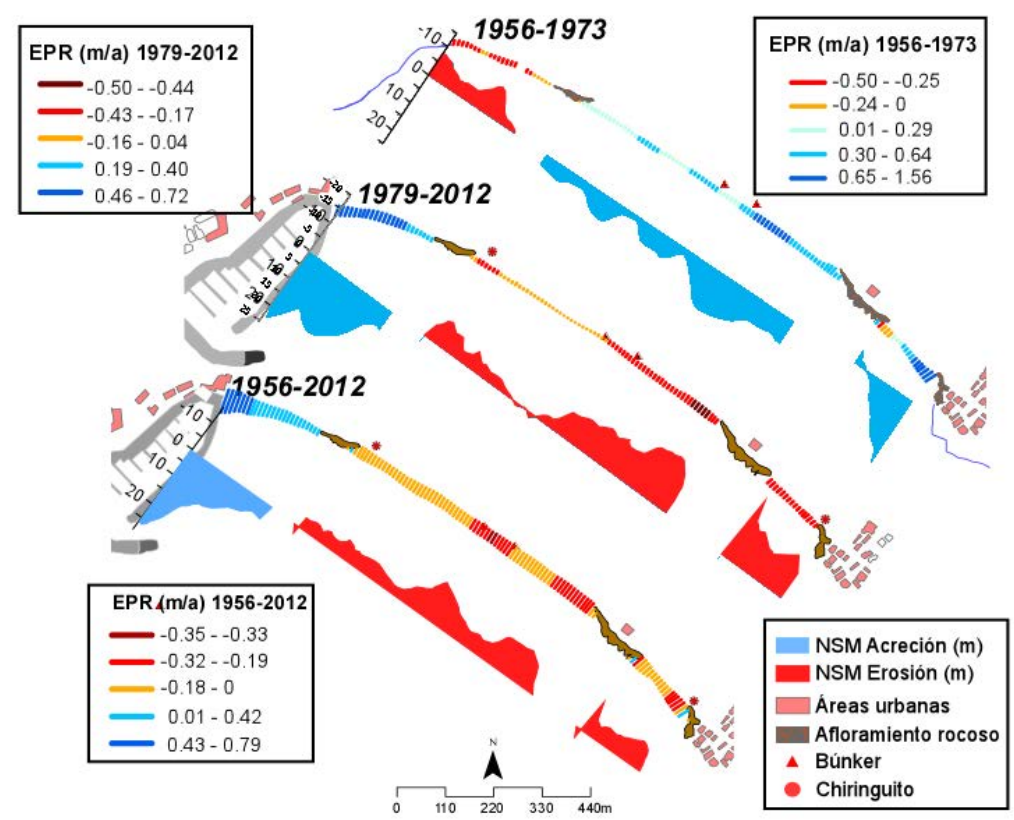

(Nótese que los valores del eje y están invertidos, para visualizar mejor los resultados. Valores positivos, significan acreción) Elaboración propia.

Figura 5. Valores de EPR, NSM, WLR y SCE para el periodo 1956-1973, 1979-2012 y 1956- 2012

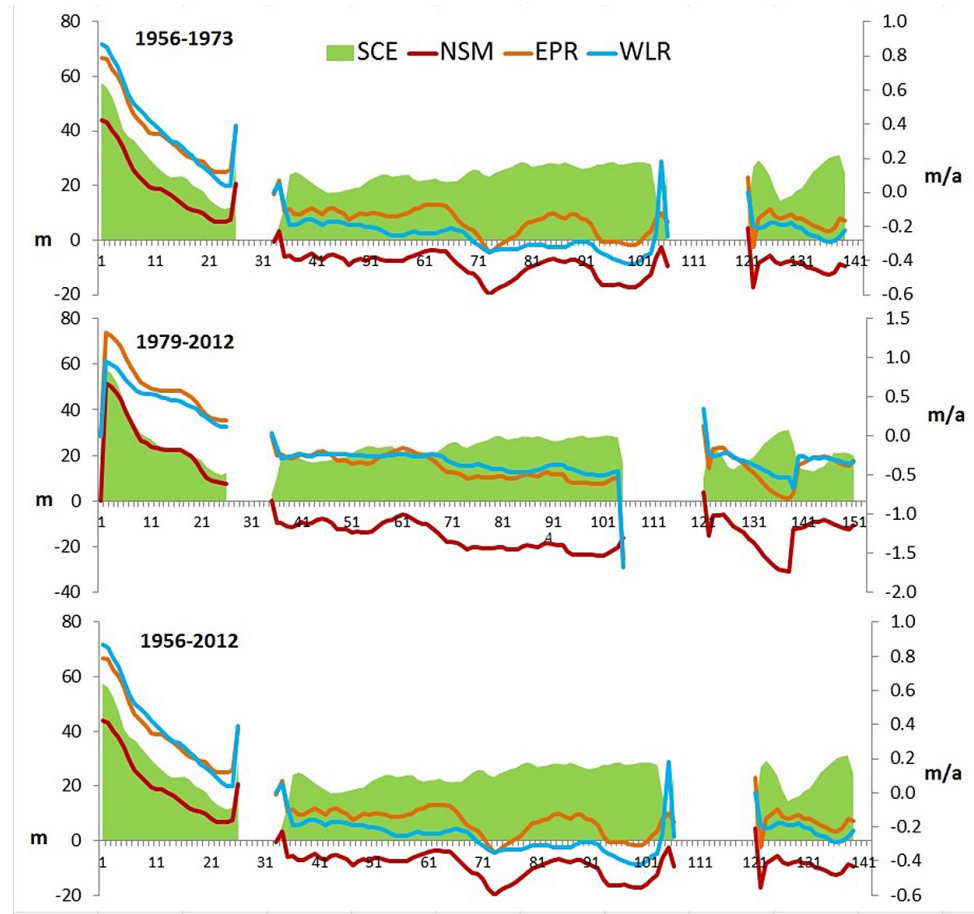

Elaboración propia. 
A partir de los datos del SCE se puede observar la diferencia entre 1956-1973 y entre 1979-2012, que muestran un incremento notable en la movilidad de la playa en el último periodo en los sectores Puerto y Central y en menor medida en el de ses Covetes (figura 7). No obstante, entre los perfiles 73 y 104, y 125 y 136 (del periodo 1956-1973), ya se manifiesta una movilidad superior al resto, que los define como los transectos más erosivos de toda la playa (figura 7).

Figura 6. Valores de EPR (m/a) y NSM (m) para el periodo entre 1979-1981 y 1981-1983

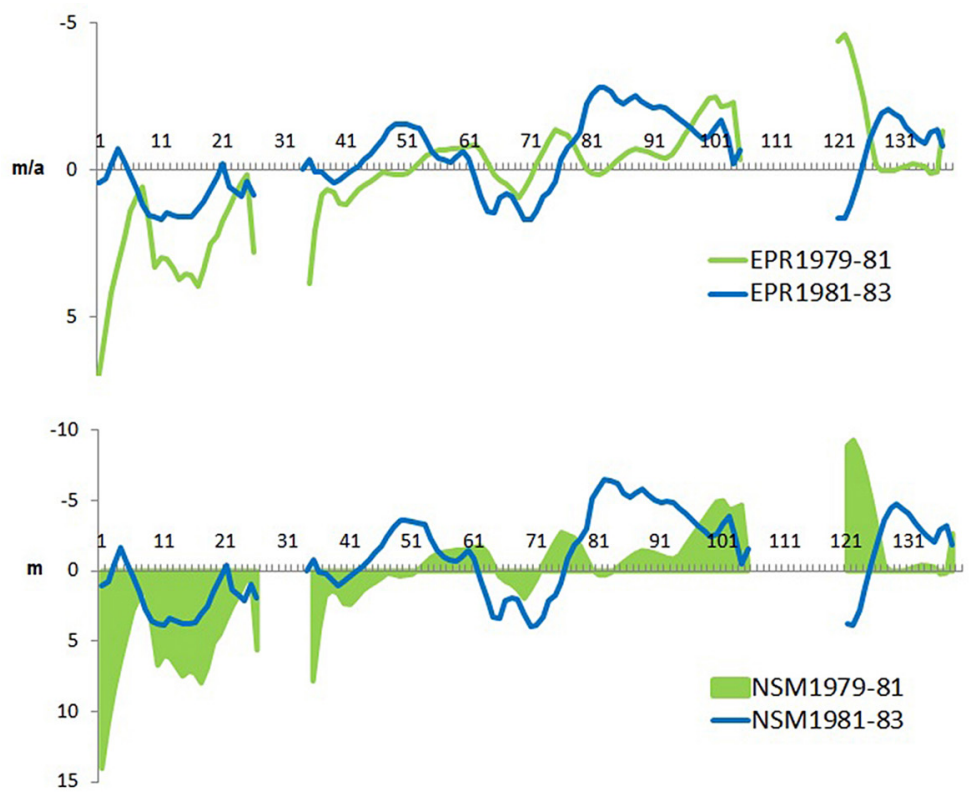

(Nótese que los valores del eje y están invertidos, para visualizar mejor los resultados. Valores positivos, significan acreción) Elaboración propia.

Figura 7. Valores de SCE (m) y NSM (m) para el periodo 1956-1973 y 1979-2012

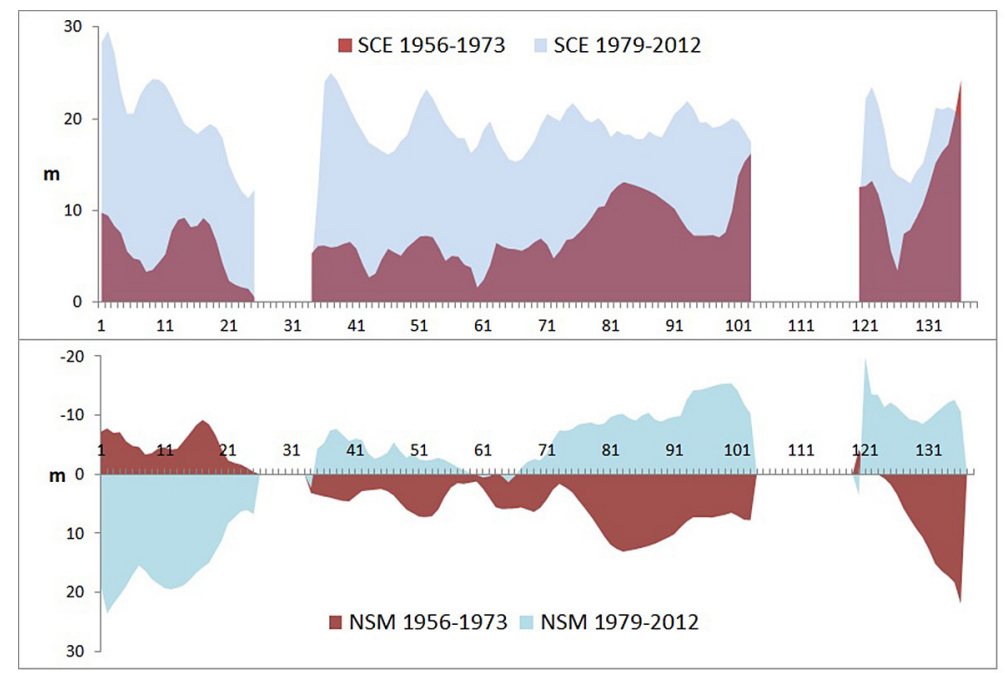

(Nótese que los valores del eje y para NSM están invertidos para visualizar mejor los resultados. Valores positivos, significan acreción) Elaboración propia.

La gráfica inferior de la figura 7, el NSM, muestra el desplazamiento de la superficie de la playa, anterior y posterior a la construcción del puerto. Se observa claramente el retroceso de la misma en los sectores Central y ses Covetes y por el contrario un crecimiento importante en el sector próximo al puerto. El 
balance global entre 1956 y 2012 es una erosión de $8.164 \mathrm{~m}^{2}$ que se sitúa exclusivamente en los sectores Central y ses Covetes (figura 4) y una acreción de $4.984 \mathrm{~m}^{2}$ en el sector del Puerto. Esto significa, que además de un trasvase de sedimento hacia el sector Puerto, este queda retenido, ya que la infraestructura actúa como trampa sedimentaria, lo que facilita los procesos erosivos en el resto de la playa. Parece claro, que el puerto deportivo influye en el balance sedimentario del conjunto, pero existen otras causas que generan otros procesos erosivos y el retroceso de la playa hacia tierra

\section{DISCUSIÓN DE RESULTADOS}

La construcción del puerto deportivo a partir de 1977 supone un cambio drástico en la dinámica sedimentaria de la playa, con importantes procesos de acreción en el sector Puerto y erosivos en el resto de la playa (figura 4).

La alteración de la dinámica sedimentaria de una playa por algún elemento antrópico, ya sea espigón o una infraestructura portuaria, implica cambios sustanciales en el transporte de sedimento. En el archipiélago balear son las instalaciones portuarias las que más han influenciado en la morfodinámica de algunas playas, centrándose fundamentalmente en la isla de Mallorca, donde tan solo el $9 \%$ de su costa está formado por materiales no consolidados (Balaguer, 2007). Actualmente existen 45 puertos deportivos distribuidos a lo largo de la costa balear (http://www.portsib.es/), algunos de los cuales tienen como característica común ubicarse, como en el caso de sa Ràpita, en los extremos de una playa.

Sin embargo, apenas se han realizado estudios para conocer el impacto de la infraestructura sobre la playa. Generalmente, se tienen datos del efecto del puerto sobre la playa debido a que el estudio se ha realizado básicamente sobre esta.

Este es el caso de la Playa de Palma (Coll, Feli de la Peña, Llabrés, Romera \& Rullán, 2008). Estos autores cuantifican con la ayuda de un SIG, un aumento de la superficie de la playa en un $26.5 \%$ entre 1956 y 1979, debido a la construcción de los dos puertos deportivos, ubicados en ambos extremos de la misma. Señalan además que estas construcciones han actuado como barreras de retención del sedimento que transporta la deriva litoral, cuya dirección es SE-NO. Esta circunstancia provocó que el crecimiento de superficie de arena, se concentrase principalmente en los extremos de la playa protegidos por los diques portuarios. Este fenómeno se corrobora con la decisión en el año 1981, al tener que modificar la bocana del puerto más septentrional, debido a los problemas de aterramiento ocasionados por la entrada constante de arena.

Posteriormente, se constató este proceso de sobre sedimentación de ambos puertos en un trabajo realizado por Gómez, Orfila, Álvarez, Cañellas \& Tintoré (2011). Estos autores, utilizando la herramienta DSAS, aportan un crecimiento en las inmediaciones de los puertos de una acreción superior a $1 \mathrm{~m} / \mathrm{a}$.

La otra infraestructura de la que se disponen datos corresponde al Puerto de Alcúdia, ubicado en el extremo norte de la bahía del mismo nombre de la costa NE de Mallorca. En un estudio elaborado por Martín y Servera (2006), pone de manifiesto el efecto trampa del puerto, ya que el sentido de la deriva litoral lo hace en dirección S-N, por lo que el sedimento queda retenido por los espigones, que fueron ampliados a partir de la década de 1980. Se estimó un crecimiento en el sector más próximo al puerto de 19.700 $\mathrm{m}^{2}$ entre los años 1981 y 2001. Posteriormente, Mir (2016) elaboró un informe para evaluar el impacto ambiental para la ampliación del puerto. El mismo se realiza con la ayuda de la herramienta DSAS y con concluye que la infraestructura facilita que el sedimento se deposite en la zona más próxima a la misma, corroborando el trabajo de Martín y Servera (2006). Los datos aportados son de un crecimiento de entre 47 y 83 m de NSM en el periodo comprendido entre 1981 y 2015. Por su parte, los datos de EPR muestran una acreción de entre 0.9 y $1.4 \mathrm{~m} / \mathrm{a}$, con un máximo de $1.7 \mathrm{~m} / \mathrm{a}$ de un transecto situado a $300 \mathrm{~m}$ del puerto.

Además del impacto ocasionado por la construcción del puerto, se han de señalar otras causas que han contribuido a la degradación y erosión del sistema playa-duna de sa Ràpita, que aparentemente son de menor importancia en comparación con la magnitud de la infraestructura, pero que sin embargo consideramos importantes.

Entre ellas está la retirada sistemática con maquinaria pesada de las acumulaciones de hojas muertas de Posidonia oceanica (conocidas como banquette) que depositadas sobre la playa, fundamentalmente a partir del otoño, son consideradas como suciedad. Esta retirada se efectúa al inicio y a lo largo de la temporada turística. Extendida por todo el archipiélago, esta práctica constituye una pérdida de sedimento 
importante. Se han estimado volúmenes de retirada entre 2.100 y $22.500 \mathrm{~m}^{3}$ de banquettes para las islas de Menorca y Formentera respectivamente a lo largo de los años 2003 y 2004 (Roig \& Martín, 2010).

Figura 8. Acopio de Posidonia oceánica depositada en la parte posterior de la playa y detalle de la cantidad de arena que se retira en su recogida con maquinaria pesada.

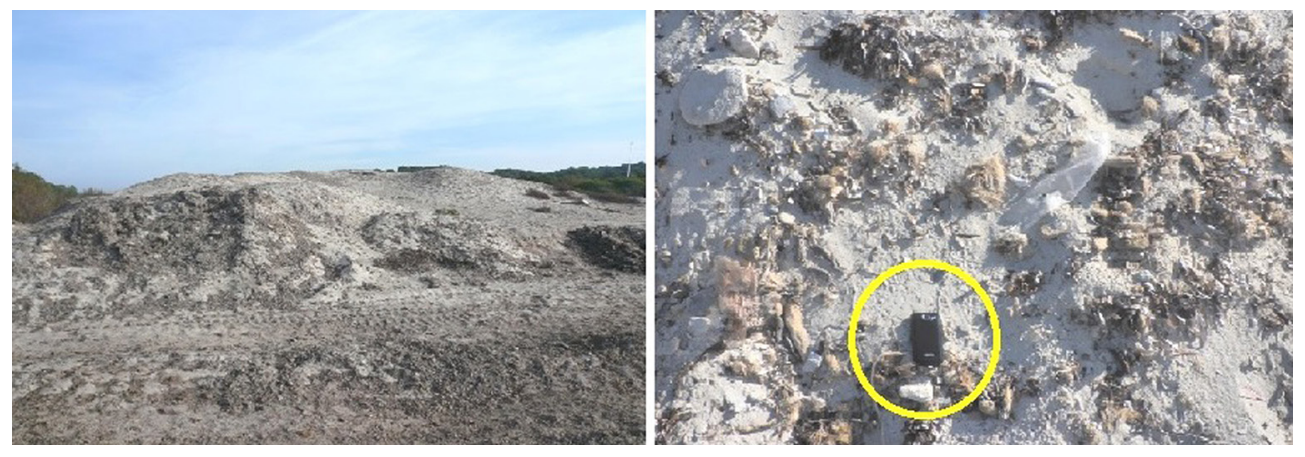

En la fotografía de la derecha, el teléfono (aprox. $10 \mathrm{~cm}$ ) nos sirve de escala visual.

Elaboración propia.

La retirada se realiza con maquinaria pesada y sin criterios técnicos ni de minimización de impactos geomorfológicos, por lo que en la operación se pueden retirar cientos de metros cúbicos de sedimento (Roig \& Martín, 2005; Simeone, 2008, Simeone \& De Falco 2013), que pueden alterar el balance sedimentario (figura 8). En los volúmenes de Posidonia oceanica estimados para Menorca y Formentera, se calculó un contenido en sedimento entre 99 y $675 \mathrm{~kg}$ de sedimento respectivamente por metro cúbico de banquette. En la Isla de Ibiza, un estudio realizado en varias playas y vertederos destinados a tal fin, obtuvo un promedio de $508 \mathrm{~kg} / \mathrm{m}^{3}$ (Roig \& Martín, 2010).

Otra de las causas que facilitan los procesos erosivos es el elevado número de visitantes en época estival, que facilita el trasiego de usuarios sobre la duna delantera. En este sentido, un estudio elaborado por Mare Nostrun (2005), señala que la frecuentación de la playa de sa Ràpita alcanzó los 2.830 visitantes en un día festivo del mes de julio de 2008 y 1.300 en un día laborable. Teniendo en cuenta que la superficie útil de playa (para esa fecha era de $34.000 \mathrm{~m}^{2}$ ), relacionada con la superficie útil por usuario $\left(15 \mathrm{~m}^{2}\right)$, tenemos que los días festivos hay una saturación del $24.9 \%$ (Mare Nostrum, 2005). Este fenómeno facilita que muchos usuarios se instalen sobre o detrás de la duna delantera, facilitando la erosión con su trasiego por la misma, eliminando la vegetación pionera y creando canales de deflación.

\subsection{Tendencias de futuro}

Uno de los objetivos planteados es poder interpretar cual puede ser la tendencia de la playa en el futuro. Para ello se analiza la recta de regresión (LRR, LSM y NSM) en varios transectos distribuidos a lo largo de la playa (figuras 9 y 10 , tabla 3).

De los 6 transectos analizados (figura 9), solamente dos presentan una tendencia de acreción (T2 y T22). Corresponden al sector Puerto, con un promedio de 0.85 y $0.11 \mathrm{~m} / \mathrm{a}$, respectivamente, a pesar de que en el periodo 1956-1973 presentaba tasas negativas (figura 10). La diferencia entre esos valores denota un contraste importante en la tasa de sedimentación en ese sector, aunque el T2 se ralentiza entre 1995-2012, podemos distinguir un cambio en la tendencia a partir del año 1995 para el T22, con un incremento en la tasa de acreción (recta de color negro). Esto puede significar un estancamiento en la mitad occidental del sector Puerto (figura 9), debido a un menor aporte de sedimento, a medida que se erosiona el resto de la playa. La figura 10, también nos muestra una disminución de valores tanto en LMS como NSM a medida que nos acercamos en el tiempo. Ambos parámetros presentan tasas negativas en el primer periodo, pasando a un incremento notable en el siguiente (1979-1995), tanto en tasas absolutas de metros (NSM), como relativas (LMS) y finalmente la disminución de las mismas en el último periodo, aunque los valores sean todos positivos, que se muestra en el reducido tamaño de las cajas, especialmente el NSM (figura 10).

Los otros cuatro transectos (T38, T70, T101 y T115), distribuidos en los sectores Central y ses Covetes, presentan como característica común la tendencia erosiva en el conjunto entre 1956 y 2012, con un rango que oscila entre -0.18 y -0.43 m/a en T115 y T101 respectivamente. Dos de los tres del sector 
Central (T70 y T101), muestran una recuperación en el último periodo, especialmente el T70, que lo hace incluso con valores positivos (figura 9).

En cambio en el periodo intermedio, 1979-1995, hay una mayor variabilidad, que también queda reflejada en la figura 10, especialmente en el sector Central. El diagrama de cajas correspondiente al LMS, muestra esa mayor variación, con valores positivos, pero el $75 \%$ restante es erosivo, con tasas de hasta $-1.28 \mathrm{~m} / \mathrm{a}$. El NSM, presenta tasas positivas de hasta $2 \mathrm{~m}$, pero es concordante con LMS, resultando igualmente un $75 \%$ erosivo. En este mismo periodo, el sector de ses Covetes, aún muestra tasas positivas de LMS, sin embargo el balance sedimentario es negativo, con valores de NSM de hasta $-4 \mathrm{~m}$, mostrando una escasa variabilidad (figura 10).

Para el periodo final, 1995-2012, la tendencia erosiva del sector Central se ralentiza y queda reflejada en el diagrama correspondiente al LMS. Lo mismo ocurre con el NSM, que muestra una menor variabilidad En cambio, en el sector de ses Covetes, todos los valores son claramente negativos (figuras 9 y 10 , tabla 3). El sector del Puerto, también muestra síntomas de ralentización, con una disminución notable en valores de NSM con respecto al periodo anterior, aunque claramente acrecionarios.

En definitiva, las tendencias muestran un comportamiento diferenciado para cada sector, siendo marcadamente negativo el de ses Covetes. Los otros dos muestran un ligero cambio en la tendencia a partir del periodo 1995-2012. Así, en el sector Puerto las tasas positivas disminuyen y en el Central las erosivas, también lo hacen.

A partir de estos datos, podría interpretarse una cierta conectividad entre los sectores Puerto y Central, condicionado por el clima marítimo, con una deriva litoral en ambos sentidos (figura 2), que facilita el intercambio de sedimento entre ambos. Sin embargo, existe una parte de ese sedimento retenido en el sector Puerto y que difícilmente se traslade al resto de la playa como ocurría antes de la construcción del puerto, que, en definitiva, es el desencadenante de la desestabilización de la playa.

Las tendencias en la evolución de la línea de costa nos permiten asegurar que el sector Puerto continuará creciendo en superficie, en mayor medida hacia la infraestructura que en el resto, como consecuencia del efecto trampa del mismo, pero a un ritmo inferior. En el sector Central, continúan los procesos erosivos, pero con una ralentización de los mismos, a pesar que entre el transecto 70 y el promontorio rocoso, las tasas de EPR son las más elevadas, alcanzando los $-0.35 \mathrm{~m} / \mathrm{a}$. Finalmente, el sector de ses Covetes, muestra los signos más erosivos de toda la playa, a pesar de su ralentización en los últimos años de este estudio.

Figura 9. Valores de EPR y recta de regresión (LRR) para diferentes transectos distribuidos lo largo de la playa

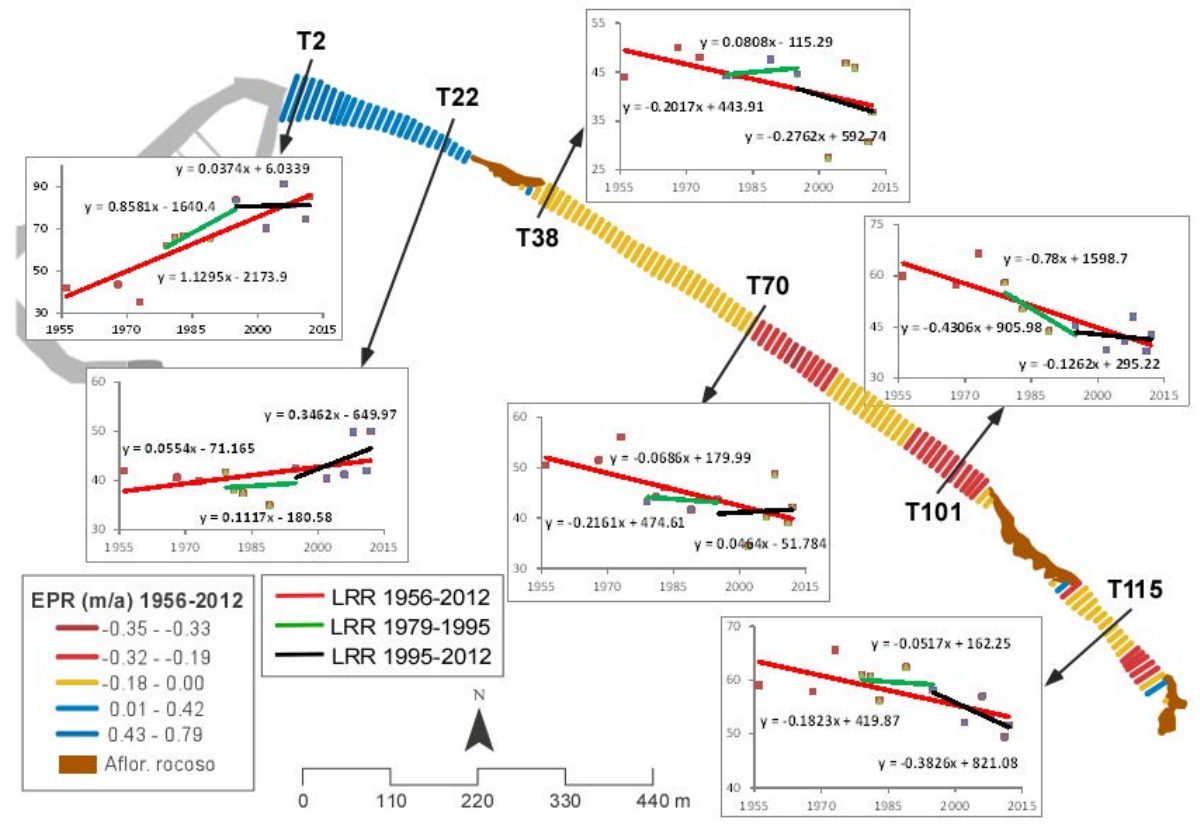

(la línea roja corresponde a la recta entre 1956-2012, la naranja entre 1979-1995 y la azul entre 1995-2012)

Elaboración propia. 
Tabla 3. Principales valores por sectores y periodo de tiempo de NSM (m) y LMS (m/a).

\begin{tabular}{|c|c|c|c|c|c|c|c|c|c|}
\hline & \multicolumn{3}{|c|}{ S. Puerto } & \multicolumn{3}{|c|}{ S. Central } & \multicolumn{3}{|c|}{ S. Covetes } \\
\hline LMS & 1956-1973 & 1979-1995 & $1995-2012$ & 1956-1973 & 1979-1995 & 1995-2012 & 1956-1973 & 1979-1995 & 1995-2012 \\
\hline No. de obs. & 25 & 25 & 25 & 73 & 73 & 73 & 18 & 18 & 18 \\
\hline Mínimo & -0.55 & -0.63 & -0.33 & -0.05 & -1.38 & -0.94 & -0.25 & -0.17 & -1.00 \\
\hline Máximo & -0.02 & 1.28 & 1.15 & 0.78 & 0.56 & 1.48 & 1.32 & 0.67 & 0.51 \\
\hline $1^{\circ}$ Cuartil & -0.42 & 0.16 & 0.36 & 0.21 & -1.17 & -0.49 & 0.07 & -0.09 & -0.60 \\
\hline Mediana & -0.27 & 0.40 & 0.56 & 0.36 & -0.36 & -0.25 & 0.50 & -0.05 & -0.49 \\
\hline $3^{\circ} \mathrm{Cuartil}$ & -0.21 & 0.62 & 0.72 & 0.44 & -0.01 & -0.03 & 0.87 & 0.17 & -0.40 \\
\hline Media & -0.30 & 0.30 & 0.51 & 0.36 & -0.50 & -0.20 & 0.48 & 0.08 & -0.44 \\
\hline Desv. Típica & 0.15 & 0.49 & 0.30 & 0.20 & 0.57 & 0.47 & 0.47 & 0.27 & 0.35 \\
\hline NSM & 1956-1973 & 1979-1995 & $1995-2012$ & 1956-1973 & $1979-1995$ & $1995-2012$ & 1956-1973 & 1979-1995 & 1995-2012 \\
\hline Mínimo & -9.20 & -3.40 & 1.63 & -0.26 & -13.58 & -8.54 & -4.24 & -2.79 & -21.44 \\
\hline Máximo & -0.41 & 21.95 & 14.89 & 13.12 & 2.83 & 2.98 & 21.93 & 0.53 & 5.65 \\
\hline $1^{\circ}$ Cuartil & -7.00 & 1.86 & 6.00 & 3.62 & -5.70 & -5.33 & 0.97 & -2.16 & -10.49 \\
\hline Mediana & -4.62 & 9.81 & 7.45 & 5.90 & -1.59 & -3.03 & 7.20 & -1.64 & -9.23 \\
\hline $3^{\circ}$ Cuartil & -3.56 & 11.38 & 8.90 & 7.33 & 0.23 & -1.61 & 14.60 & -1.04 & -7.64 \\
\hline Media & -5.01 & 8.01 & 7.32 & 6.10 & -3.23 & -3.14 & 7.81 & -1.53 & -9.09 \\
\hline Desv. Típica & 2.42 & 6.45 & 2.53 & 3.30 & 4.88 & 2.91 & 7.88 & 0.85 & 4.99 \\
\hline
\end{tabular}

Valores negativos significan erosión

Elaboración propia.

Figura 10. Diagrama de cajas mostrando las variables LMS y NSM

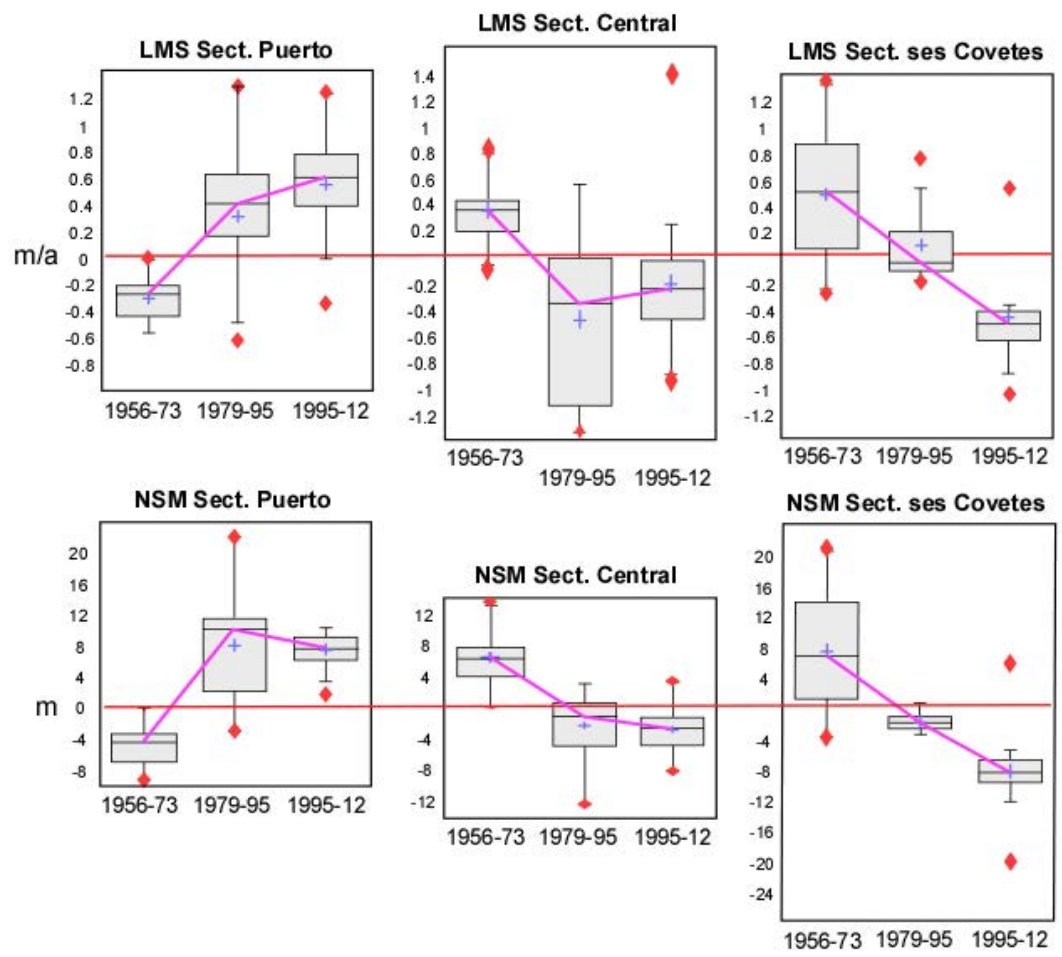

(La línea horizontal roja es cero, valores negativos significa erosión). La caja comprende el rango entre $1^{\text {er }}$ y $3^{\text {er }}$ cuartil, la mediana ( $2^{\circ}$ cuartil) es la línea negra y la media la cruz azul. Los bigotes señalan los valores máximos y mínimos. Los puntos rojos son anomalías (outliers). Elaboración propia.

\section{CONCLUSIONES}

La evolución histórica de la posición de la línea de costa es importante para poder evaluar los efectos espaciales de la dinámica litoral. La utilización de una herramienta denominada Digital Shoreline Analysis System [DSAS], dentro del marco de un SIG, permite el cálculo estadístico a partir de múltiples posiciones de la línea de costa a lo largo del tiempo. 
Los parámetros estadísticos utilizados (EPR, NSM, SCE, LRR, WLR y LMS), nos han permitido conocer el comportamiento histórico de la línea de costa en la playa de sa Ràpita (Sur de Mallorca) en un periodo comprendido entre 1956 y 2012. Inicialmente, la morfodinámica de la playa presentaba una deriva litoral en ambos sentidos como consecuencia de la bimodalidad de su clima marítimo.

Sin embargo, la playa se ha visto fuertemente alterada como resultado de la construcción de un puerto deportivo en su extremo NO a partir del año 1977, que ha alterado el transporte de sedimento por acción de la deriva litoral, facilitando la sedimentación en el sector del Puerto y la erosión en el resto de la playa.

Hasta 1973, último fotograma anterior a la construcción del puerto, el análisis de la línea de costa presentaba acreción en el sector Central $(0.35 \mathrm{~m} / \mathrm{a})$ y ses Covetes $(0.5 \mathrm{~m} / \mathrm{a})$ y erosión en el sector Puerto (-0.31 m/a). A partir de 1979 (primer fotograma posterior a la construcción), la tendencia se invierte y el sector próximo al puerto comienza a ganar superficie $(16.28 \mathrm{~m})$, mientras que el resto de la playa se erosiona (-6.34 y -11.28 m en los sectores Central y ses Covetes respectivamente). Esta tendencia general continúa hasta la actualidad, aunque parece disminuir la acreción en el sector próximo al puerto y los procesos erosivos en el resto de la playa, presumiblemente como consecuencia de una estabilización en el trasvase de sedimento entre los diferentes sectores de la playa y un menor aporte del mismo como debido al déficit sedimentario acumulado a lo largo de las últimas décadas.

En definitiva, la construcción del puerto deportivo a partir de 1977 supone un cambio drástico en la dinámica sedimentaria de la playa, con importantes procesos de acreción en el sector Puerto y erosivos en el resto de la playa. A ello ha contribuido también la retirada masiva de hojas de Posidonia oceanica (banquettes) acumuladas sobre la playa y un uso masivo de usuarios en época estival que han alterado la duna delantera con su trasiego sobre la misma.

La situación planteada desde la construcción del puerto supone un trasvase de sedimento por la deriva litoral hacia ese sector procedente del resto de sectores, sedimento que ya no se distribuye hacia el resto de la playa, si no que queda retenido aumentando su superficie. Finalmente, este trabajo ha permitido validar el análisis realizado por Servera y Martín (1996), es decir, pérdida de superficie de playa en el sector de ses Covetes, fuertes y acelerados procesos erosivos en la mitad este del sector Central, esto es, entre el afloramiento rocoso y los bunkers y altos índices de acreción en el sector Puerto.

\section{REFERENCIAS}

Albertí, J. B. (1998). La defensa de Mallorca, Ibiza y Formentera durante la II Guerra Mundial. Inédito. Palma.

Arias, C. E. (2003). Spatio-temporal analysis of Texas shoreline changes using GIS technics. PhD Thesis, Texas A\&M University. Texas.

Balaguer, P. (2007). Inventari cuantitatiu de les costes rocoses de Mallorca. En Pons, G. X. y Vicens D. (Eds.), Geomorfologia li toral i Cuaternari. Homenatge a Joan Cuerda Barceló (pp. 201-230). Palma: Monografies de la Societat d'Història Natural de les Balears 14.

Balaguer, P., Vallesir, J., Vizoso, G., Ruíz, M., Cañellas, B. y Tintoré, J. (2007). Atlas de Sensibilidat del Litoral de les Illes Balears. Conselleria d'Interior: Govern de les Illes Balears.

Boak, E. y Turner, I. L. (2005). Shoreline definition and detection: a review. J. Coastal. Research 21(4), 688-703.

Cancemi, G. \& Duron, K., (2008). Erosion du littoral et suivi des banquettes de Posidonie sur les plages de Corse. DIREN Corse / E.V.E.Mar.

Cañellas, B. (2010).Long-term extreme wave height event in the Baleareic Sea: characterization, variability and prediction. Tesis Doctoral, Universitat de les Illes Balears. Palma.

Catalao J., Catita C., Miranda J. \& Dias J. A. (2002). Photogrammetric analysis of coastal erosion in the Algarve (Portugal). Geomorphologie, 2, 119-126.

Coll, M. A., Feli de la Peña, J. F., Llabrés, N., Romera, J. y Rullán, B. (2008). Evolución de la línea de costa de la playa de Palma-Arenal (1956-2004). Territoris, 7, 193-2002.

Costa Balear Sostenible. Recuperado de http://www.costabalearsostenible.com

Cowart, L., Walsh, J. P. \& Corbett, D. R. (2010). Analyzing Estuarine Shoreline Change: A Case Study of Cedar Island, North Carolina. J. Coastal Research, 26(5), 817-830. 
Coyne, M.A., Fletcher, C. H. \& Richmond, B. M. (1999). Mapping coastal erosion hazards in Hawaii: observations and errors. J. Coastal Research SI, 28, 171-184.

Crowell, M., Leatherman, S. P. \& Buckley M. K. (1991). Historical shoreline change: error analysis and mapping accuracy. J. Coastal Research, 7, 839-852.

De Falco, G., Baroli, M., Simeone, S. \& Pergalini, G. (2002). La rimozione della Posidonia dalle espiagge: conseguenze sulla stabilità del litorali. Proggeto ARENA, Oristano, Italia. Fondacione IMC.

Del Río, L. y Gracia, F. J. (2013). Error determination in the photogrammetric assessment of shoreline changes. Nat. Hazards, 65, 2385-2397.

Dolan, R., Hayden, B. P., May, P. \& May, S. K. (1980). The reliability of shoreline change measurements from aerial photographs. Shore and Beach, 48(4), 22-29.

Dolan, R., Fenster, M. S. \& Olmes, J. (1991). Temporal analysis of shoreline recession and accretion. J. Coastal Research, 7(3), 723-744.

Douglas, B. C. \& Crowell, M. (2000). Long-term shoreline position prediction and error propagation. J. Coastal Research, 16, 145-152.

Elginoz, N., Kabdaslit, M. S. \& Tanik, A. (2011). Effects of Posidonia Oceanica Seagrass Meadows on Storm Waves. J. Coastal Research SI, 64, 373-377.

Elliot, I. \& Clarke, D. (1989). Temporal and spatial bias in the estimation of shoreline rate-of-change statistics from beach survey information. Coastal Management, 17, 129-156.

Fletcher, C., Rooney, J., Barbee, M., Lim, S. \& Richmond, B. M. (2003). Mapping shoreline change using digital ortophotogrametry on Maui, Hawaii. J. Coastal Research SI 38, 106-124.

Gacia, C. y Duarte, C. M. (2001). Sediment Retention by a Mediterranean Posidonia oceanica Meadow: The Balance between Deposition and Resuspension. Estuarine, Coastal and shelf Sci., 52, 505-514.

Genz, A. S., Fletcher, C. H., Dunn, R. A., Frazer, L. N. \& Rooney, J. (2007). The predictive accuracy of shoreline change rate methods and alongshore beach variation on Maui, Hawaii. J. Coastal Research 23(1), 87-105.

Gómez, L., Orfila, A., Cañellas, B., Álvarez, A., Méndez, F. J., Medina, R. y Tintoré, J. (2007). Morphodynamic classification of sandy beaches in low energetic marine environment. M. Geology, $242,235-246$.

Gómez, L., Orfila, A., Cañellas, B., Álvarez, A. y Tintoré, J. (2011). Evolución de la línea de costa en una playa urbana (S'Arenal de Mallorca): intervención antrópica y dinámica natural. En Montoya, I., Rodríguez, I, y Sánchez, M. J. (Eds.), Avances en Geomorfología Litoral. Actas de las VI Jornadas de Gemorfología Litoral (pp. 71-74). Tarragona.

Govern de les Illes Balears. Infraestructura de Dades Espacials de les Illes Balears [IDEIB]. Recuperado de http://www.ideib.cat

Govern de les Illes Balears. PortsIB. Recuperado de http://www.portsib.es

Hughes, M. L., McDowell, P. F. \& Marcus, W.A. (2006). Accuracy assessment of georectified aerial photographs: implications for measuring lateral channel movement in a GIS. Geomorphology, 74, 1-16.

Infantes, E., Terrados, J., Orfila, A., Cañellas, B. y Álvarez, A. (2009). Wave energy and upper depth limit distribution of Posidonia oceanica. Botanica Marina 52, 419-427.

Jaume, C. y Fornós, J. J. (1992). Composició i textura dels sediments de les platges del litoral mallorquí. Boll. Soc. Hist. Nat. Balears, 35, 93-110.

Jones, B.M., Hinkel, K. M., Arp, C. D. \& Eisner, W. R. (2008). Modern erosion rates and loss of coastal features and sites, Beaufort Sea coastline, Alaska. Arctic 61,(4), 361-372.

Kumar A. \& Jayappa, K. S. (2009). Long and short-term shoreline changes along Mangalore Coast, India. Int. J. Environ. Research, 3, 177-188.

Manca, E., Cáceres, I., Alsina, J.M., Stratigaki, V., Townend, I. \& Amos, C.L. (2012). Wave energy and wave-induced flow reduction by full-scale model Posidonia oceanica seagrass. Continent. Shelf Research, 50-51, 100-116. 
Mare Nostrum (2005). Detecció i anàlisi de les presions i impactes derivats de l'ús públic en l'àmbit PORN. (Pla d'ordenació dels recursos naturals) de Es Trenc-Salobrar de Campos (Mallorca, Illes Balears) (Inèdit). Tarragona: Conselleria de Medi Ambient. Direcció General de Biodiversitat.

Martín, J. A. y Servera, J. (2006). Erosión costera del sector comprendido entre s'Oberta del Gran Canal y el Puerto de Alcúdia (NE de Mallorca). En Pérez, A. y López, J. (Eds.), Geomorfología y Territorio: Actas de la IX Reunión Nacional de Geomorfología (pp. 101-105). Santiago.

Martín, J. A., Roig, F. X., Rodríguez, A. y Pons, G. X. (2010). Evolució de la línia de costa de la platja de es Trenc. En Mayol, J., Muntaner, L. y Rullán, O. (Eds.), Homenatge a Bartomeu Barceló Pons, Geògraf (pp. 423-440). Palma: L'Arjau.

Ministerio de Fomento. Puertos del Estado. Recuperado de http://www.puertos.es

Ministerio de Fomento. Infraestructura de Datos Espaciales de España. Recuperado de http://www.idee.es/

Mir, M. (2016). Estudio y análisis espacio-temporal de la línea de costa y la playa limítrofe al puerto deportivo Alcudiamar. Alcudiamar S.L. Palma. Informe inédito.

Moore, L.J. (2000). Shoreline mapping techniques. J. Coastal Research, 16(1), 111-124.

Morton, R. A., Miller, T. A. \& Moore, L. J. (2004). National assessment of shoreline change: Part 1. Historical shoreline changes and associated coastal land loss along the US Gulf of Mexico. USGS OFR, 2004-1043.

Nordstrom K.F. (2000). Beaches and Dunes of Developed Coasts. Cambridge, Inglaterra: Cambridge University Press.

Ojeda, J. (2000). Métodos para el cálculo de la erosión costera. Revisión, tendencias y propuestas. Boletín de la Asociación de Geógrafos Españoles, 30, 103-119.

Ojeda, J., Vallejo, I., Hernández, L. y Álvarez, J. (2007). Fotogrametría digital y LIDAR como fuentes de información en geomorfología litoral (marismas mareales y sistemas dunares): el potencial de su análisis espacial a través de SIG. Boletín de la Asociación de Geógrafos Españoles, 44, 215-233.

Pajak, M. J. \& Leatherman, S. P. (2002). The high water line as shoreline indicator. J. Coastal Research $18(2), 329-337$.

Pérez, A., Pires, A., Freitas, L., Rodrigues, C. y Chaminé, H. I. (2011). GIS mapping and shoreline change analysis along the rocky coast of Galicia (NW Spain): preliminary approach. In Institution of Civil Engineers (Eds.), 7th Conference on Coastal Management, Innovative Coastal Zone Management: Sustainable Engineering for a Dynamic Coast, Belfast (pp. 262-263). London: ICE Publishing.

Ratsimandresy, A. W., Sotillo, M. G., Carretero, J. C, Álvarez, E. \& Hajji. H. (2008). A 44-year highresolution ocean and atmospheric hindcast for the Mediterranean Basin Developer within the HIPOCAS Project. Coast. Eng., 55, 825-826.

Rodríguez, I., Montoya, I., Sánchez, M. J. y Carreño, F. (2009). Geographic Information Systems applied to Integrated Coastal Zone Management. Geomorphology, 107, 100-105.

Roig, F. X. y Martín, J. A. (2005). Efectos de la retirada de bermas vegetales de Posidonia oceánica sobre las playas de las Islas Baleares: consecuencias de la presión turística. Investigaciones Geográficas de México 57, 39-52.

Roig, F. X. y Martín, J. A. (2010). Criteris de gestió de restes acumulades de Posidonia oceánica a les platges d'Eivissa per minimitzar l'impacte geoambiental. Eivissa. Consell Insular d'Eivissa.

Ruggiero, P., Kratzmann, M. G., Himmelstoss, E. A., Reid, A., Allan, J. \& Kaminsky, G. (2012). National Assessment of Shoreline Change: Historical Shoreline Change Along the Pacific Northwest Coast. Open File Report 2012-1007. US Geological Survey.

Sánchez, J. F., Sánchez, V. y Memos, C. D. (2011). Wave attenuation due to Posidonia oceanica meadows. Journal of Hydraulic Research, 49, 503-514.

Servera, J. y Martín, J. A. (1996). Análisis y causas del retroceso de la línea de costa en s’Arenal de sa Ràpita (Mallorca). En A. Grandal, y J. Pagés (Eds.), IV Reunión de Geomorfología, O Castro (A Coruña) (pp. 877-890). A Coruña: Cadernos do Laboratorio Xeolóxico de Laxe.

Simeone, S. (2008). Posidonia oceanica banquettes removal: sedimentological, geomorphological and ecological implications. Tesis Doctoral, Università degli Studi di Viterbo. Vitervo. 
Simeone, S. \& De Falco, G. (2013). Posidonia oceanica banquette removal: sedimentological, geomorphological and ecological implications. J. Coastal Research, S.I., 65, 1045-1050.

Solomon, S.M. (2005). Spatial and temporal variability of shoreline change in the Beaufort-Mackenzie region, Northwest Territories, Canada. Geo-Marine Letters, 25(2-3), 127-137.

Stratigaki, V., Manca, E., Prinos, P., Losada, I. J., Lara, J. L., Sclavo, M., ... \& Sánchez-Arcilla, A. (2011). Large-scale experiments on wave propagation over Posidonia oceanica. J. Hydrau. Research, 49 (sup1), 31-43.

Terrados, J. y Duarte, C. M. (2000). Experimental evidence of reduced particle resuspension within a seagrass (Posidonia oceanica L.) meadow. J. Experim. Marine Biology and Ecology, 243, 45-53.

Thieler E. R. \& Danforth W. W. (1994). Historical shoreline mapping (1): improving techniques and reducing positioning errors. J. Coastal Research, 10(3), 549-563.

Thieler, E. R., Himmelstoss, E. A., Zichichi, J. L., \& Ergul, A. (2009). Digital Shoreline Analysis System (DSAS) version 4.0. An ArcGIS extension for calculating shoreline change. U.S. Geological Surve9y Open-File Report, 2008-1278.

Vicens, D., Morey, B. y Pons, G.X. (2006). El Pleistocè superior marí de la badia de Campos (Sa Ràpita - Es Trenc, Mallorca, Mediterrània occidental. Boll. Soc. Hist. Nat. Balears, 49, 123-136.

Virdis, S., Oggiano, G. \& Disperati, L. (2012). A Geomatics Approach to Multitemporal Shoreline Analysis in Western Mediterranean The Case of Platamona-Maritza Beach (Northwest Sardinia, Italy). J. Coastal Research, 28 (3), 624-640.

Wright, L.D. \& Short, A.D. (1983). Morphodynamics of beaches and surf zones in Australia. En P. D. Komar (Ed.), Handbook of Coastal Processes and Erosion (pp. 35-64). , Florida: CRC Press.

Zhang, Y. \& Grassle, J.F. (2002). A portal for the Ocean Biogeographic Information System. Oceanologica Acta, 25 (5), 193-197. 